\title{
Reduction the Effect of Mobility in Link Transmitting Using Efficient DSR Route Cache for MANETs
}

\author{
Ayoob A. Ayoob, Norrozila Sulaiman, Muamer N. Mohammed, and Ghaidaa M. Abdulsahib
}

\begin{abstract}
Communication between mobile users is becoming more popular than ever before due to the recent technological advances in wireless communication devices. Ad hoc networks are generally closed in that, it's a collection of two or more devices equipped with wireless communications and networking capability; they do not connect to the Internet and are typically created between participants. This means that a formed network can be de-formed on-the-fly without the need for any system administration. Many routing protocol methods have been proposed in Mobile Ad-Hoc Network but still the challenges are to improve the routing performance. The DSR (Dynamic Source Routing) routing protocol dependent on the cache memory for every node to store the routing path from source to destination. This paper presented a new algorithm using DSR routing cache technique to improve the routing between mobile nodes to reduction the effect of mobility in link transmitting that can solve link broken problem. The simulation results show a low delay, stable link in routing and better performance in terms of Route Discovery Time. It indicates that DSR cache outperforms DSR standard and had a high quality of packet delivery.
\end{abstract}

Index Terms-Ad hoc networks, broadcasting algorithm, routing protocols, DSR, link reliability.

\section{INTRODUCTION}

The strategy of route cache in DSR protocol has been proposed in 1998 [1]. Which is used to store a route that is learned from the source node and to avoid unnecessary route discovery operation in each time the data packet is to be transmitted [2]. The reason to restart of the route discovery mechanism in reactive on demand routing protocols is very costly in term of delay, bandwidth consumption and battery power, due to flooding of the network, whereas can cause a high delay before the first data packet sent. Therefore, the main performance of DSR depends on efficient implementation of route cache. The effect a route cache on the performance of DSR observation the majority of route reply packet (REPP) based on route and only of 59 percent of Route Reply packets (RREP) carry correct routes as well as, the discovery that even (REPP) packets from the destination are not 100 percent correct, since the route may deteriorate while (REPP) packet sent back to the source node. Therefore, route maintenance is very important for all the reactive protocols with the route cache.

The route cache is a major approach to reduce the flooding in the network by avoiding route discovery operation as much

Manuscript received January 8, 2014; revised May 30, 2014.

The authors are with the Faculty of Computer Systems \& Software Engineering, University Malaysia Pahang, Malaysia (e-mail: aiobaziz@gmail.com, norrozila@ump.edu.my, muamer.scis@yahoo.com, gh961@yahoo.com). as possible so; the route cache is the essential component in DSR protocol. The route cache may be containing the stale routes which can affect for the performance of DSR protocol. The stale routes in the route cache of DSR protocol may take several adverse effects such as long delay, packet loss.

Route cache can be divided to the three different strategies cache structure, cache capacity and cache timeout as shown in Fig. 1. [3], [4].

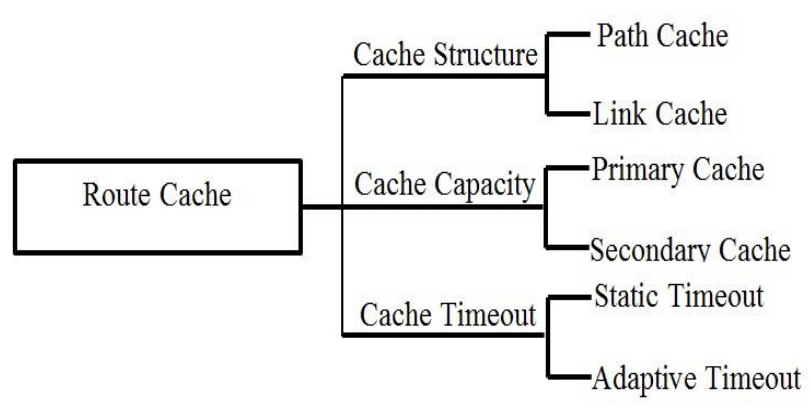

Fig. 1. Route cache strategies.

\section{THEORETICAL BACKGROUND}

There are three main problems with the current cache in DSR protocol, No Expire, rapid palliation and Incomplete Error Notification [5]. Depend on this problem they have developed three of mechanism specifically timer based cache, Wider Error Notification and negative cache. The speed and size of the RERR packets lead to route error propagation of the route that transmitted as broadcast packets at the MAC layer.

When a node receives the RERR packet that include link failure information, the node updates its route cache, therefore, that all source route that include link failure information is truncated at the point of failure. The Adaptive link cache procedure can remove the stale route in the cache of the DSR and compare it with the path of cache structure [6]. The adaptive link cache scheme is a combination to link of cache and the adaptive of timeout policy. The major concern of using the Adaptive cache Scheme is to track the optimal link lifetime with the differentiator of the node's mobility level situation. According to hi result shows that, with high load traffic in the network, the adaptive Link Cache mechanism outperforms the path cache as well as the stale route are removed from the cache.

A distributed adaptive cache Update Algorithms Proposed in [7], the major objective of this algorithm is to update the route cache of the DSR by using the proactive cache update instead of an adaptive timeout mechanism in a link cache structure to remove the stale route in a coach as well as to collect the extra information about haw routing information 
distributed through the network. In this algorithm four fields which added to the path cache structure in each node whereas each route the node maintains of information about this fourfold. The first and second fields are representing a source and destination node for the current route; the third field represents the amount of data packets that delivery to the destination node by using the current route to know haw routing information is synchronized among the entire node in the route. Finally, the field for which roams "routing table" which represents each node maintains the routing information about which neighbor have learned for this route.

"Active Packet" proposed to solve the problem stale route in cache route in DSR protocol [8]. This way depends on the link cache structure, which the active packet roams around the network, and collects routing information that has obtained by network topology. This way has two phases: the first phase updating the route cache and the second stage is gathering all the information about the network by checking a payload of active packet. In [9], proposed Tiding Active Packets (TAP) to improve the active packet method. The TAP compare of an Active Packet and Route Error RERR flooding to update the stale route in the cache of DSR protocol [10].

\section{A. Routing Protocol}

Routing is the act of carrying a piece of information from a source to a destination in an inter-network. There is an encounter of a minimum of one intermediate node inside the internet works in this process. Since the routing was already employed in the networks in the wake of the 70s, this concept is no longer a novelty in the field of computer science However, this concept has slowly been gaining popularity from the middle of the 1980s [10], as the earlier networks, despite being less complicated and functioning in homogeneous environments; high end and large scale internetworking strives in the most updated development. Fundamentally, the routing concept deals with two activities: firstly, making sure that the routing paths are optimal and secondly, moving the information groups or more specifically termed as packets along and across an internetwork.

The latter concept is termed packet switching which is very easy to understand, and the path determination can possibly become rather complicated. Routing protocols adopt several metrics for calculating the best path before the packets are sent to their intended destination. These metrics is a standard measurement using a number of hops, normally used by the routing algorithm to decide on the optimal path that should be used by the packet to its destination. The path determination process suggests that the routing algorithms kick-start and retain the routing tables, which have the entire route information for the packet that varies across the routing algorithms. Routing tables contain a wide range of information generated by the routing algorithms [11], [12]. Most common entries emerging in the routing table come in the form of the IP address prefix and the next hop. Routing table's Destination/next hop associations suggests to the router that a destination can be reached in an optimal manner by having the packet sent to the router, at the same time representing the "next hop" on its way to the final destination, and the IP -address prefix searches for a set of destinations for which the routing entry is valid.

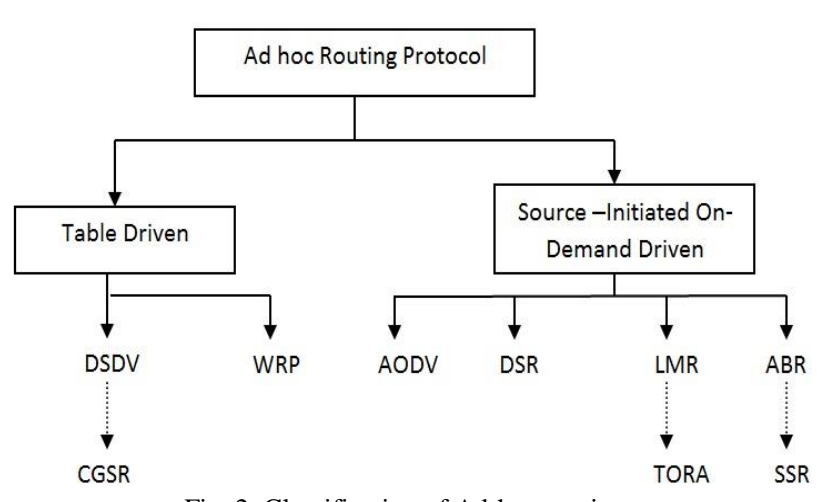

Fig. 2. Classification of Ad-hoc routing.

This works the same way with the network segments in the dynamic routing. According to the routing strategy, the routing protocols are Table-driven and source-initiated, and they rely on the network structure [13]. There are classified as flat routing, hierarchical routing and geographic position assisted routing Both the Table-driven and source initiated protocols are placed under the flat routing [14], as shown in Fig. 2.

\section{B. Dynamic Source Routing (DSR)}

DSR is a protocol that had been introduced for routing in mobile Ad-hoc networks and brought forth for MANET [15]. In brief, the nodes deliver a ROUTE REQUEST message, where all nodes which get this message will be set in the source route and forwarded to their neighbors, unless they have received the same request previously. If the receiving node has a route to the destination, the request is not forwarded, but a REPLY message informing about the full source route is sent. The answer may be committed along the source route reversibly or a ROUTE REQUEST is issued. Including the route return to the source. If the former is not regarded to be possibly due to asymmetrical connections. ROUTE REPLY messages can be provoked by ROUTE REQUEST messages. After receiving several routes at most, the source picks out the best (by defaulting the shortest), having it stored, and messages sent through the path.

The better the route metrics (number of hops, delay, bandwidth, or other criteria) and the faster the REPLY reaches the source, the higher the preference granted to the route and, the longer its stay will be in the cache. When a ROUTE REPLY arrives soon after a ROUTE REQUEST is sent, this may highlight the existence of a short path since the nodes are usually required to wait for a time to correspond to the length of the route they can advertise, before having it posted. This is done to overcome abundant replies [16]. If the connection fails, the node that is not able to forward the packet to the next node will present an error message to the author. Routes that have broken links can be rescued by taking an optional partial path that has no bad link.

The former advantage Dynamic Source Routing (DSR) protocol that is using source routing, also it is an on-demand protocol which node allow to find out a route over network dynamically [17]. The good idea behind use source routing back to the backed headers of DSR has a complete list of nodes duration they will sink to reach its distance. Accordant of the resulted there is no mechanism of route discovery packet of broadcasting in DSR. This will bring down the 
overhead bandwidth network. If there were a better route, then the node will update their route cache. The DSR has two modes of processing [8], [12] route discovers and route maintained.

Fig. 3 shows the initiate of Route Discovery in which a node $\mathrm{S}$ transmits a Route Request message attempting to discover a route to node $\mathrm{D}$. Then, all nodes will receive within wireless transmission range of $\mathrm{D}$. Each Route Request message identifies the source and destination of the Route Discovery. This route record is initialized to an empty list by the source of the Route Discovery.

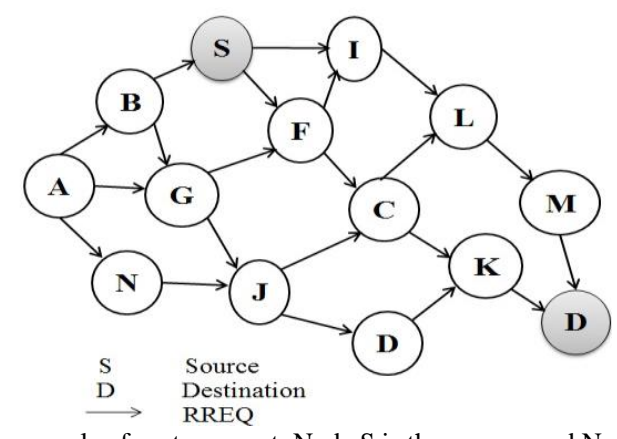

Fig. 3. An example of route request: Node $S$ is the source, and Node D is the destination.

When another node receives a Route Request as a destination of the Route Discovery, it returns a Route Reply message to the source, giving a copy of the accumulated route record from the Route Request; then the source will caches this Route Reply and use it to send subsequent packets to this destination [13].

Otherwise, if this node receiving the Route Request has recently seen from another Route Request message, this source bearing this same request ID, or if it finds that its own address is already listed in the route record then, it discards the request. Otherwise, this node will add its own address to the route record and propagates it by transmitting it as a local broadcast packet5, as shown in Fig. 4. Finally, it avoids routing loops easily because the complete route is determined by a single node instead of making the decision hop-by-hop.

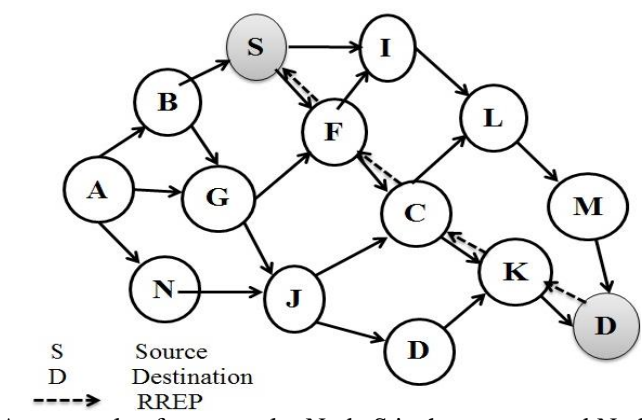

Fig. 4. An example of route reply: Node $\mathrm{S}$ is the source, and Node D is the destination.

\section{DSR Route Cache Protocol}

The main problem was in cache route due to the DSR protocol depend on the cache route to route the packet in the network, one of this problem is cache route size because the route cache size will be a large with allots of a cache records which hold the whole of the route. Therefore, when a source node needs to send a packet to a destination node in the first will check the route cache to find the best route which can be used to send a packet to the destination. The problem of broken route it happened the speed of nodes; Battery is empty, problem of route order and transmission out of range. Therefore, when the new route appended to the route cache where a new route will add to the end of the cache, therefore, the node will pick the old route to send a packet which may be broken. To resolve this problem we propose the new algorithm that alters and modify the route cache which to:

1) Solve the broken off route (stole routes).

2) The search time of route (the old and new routes).

3) Problem size of the cache.

\section{THE PROPOSED OF ALGORITHM}

In the proposed new algorithm, we add new mechanisms (route status checker, route order) and the new route of cache structure (Master route cache and Index Route Cache).

\section{A. The New Route of Cache Structure}

The cache of DSR protocol will be divided for two sub-caches: the first cache part called (Master Route Cache) which saves information about source node, the numbers of hops, the statue of the route and destination node. The second cache part called (Route index) which save all the indexes of the route and the route from the source node to the destination node. The Master Route cache will be instructed based on the shortest path (the number of hope), the newest route which will provide by a route discovery mechanism. The route discovery and the route maintenance will as propose in a protocol draft. Therefore, the proposed of version suggest changing the structure of the cache of node route only. Table I represents the proposed of structure for a node in DSR which mean every node in the network will use this new structure for saving the data about neighbors. Fundamentally, all routes are instructed depending on the new-short route.

\begin{tabular}{|c|c|}
\hline $\begin{array}{l}\text { Sub cache one Master } \\
\text { Route Cache }\end{array}$ & $\begin{array}{l}\text { Sub cache tow Index } \\
\text { route cache }\end{array}$ \\
\hline $\begin{array}{ll}- & \text { Source node } \\
\text { - } & \text { Number of hops } \\
\text { - } & \text { Route status } \\
\text { - } & \text { Destination node }\end{array}$ & $\begin{array}{l}\text { - The index of the Route } \\
\text { - The route from the } \\
\text { source to } \\
\text { destination }\end{array}$ \\
\hline
\end{tabular}

\section{B. DSR Cache Algorithm}

\section{1) The initialize process}

Start of simulation to identify of environment.

Each node in the network will initialize its cache, accordance as its source nodes and the others are destination nodes.

Enter the source name in the source node field.

Enter the destination name in a destination field.

Enter the number of hops in a hops field.

Enter the link status $(0=$ broken, $1=$ active $)$. But in beginning all links which supposed to be active.

Enter the serial number as the route index.

Enter the full route in the route field. 
Fig. 5 shows the initialization process.
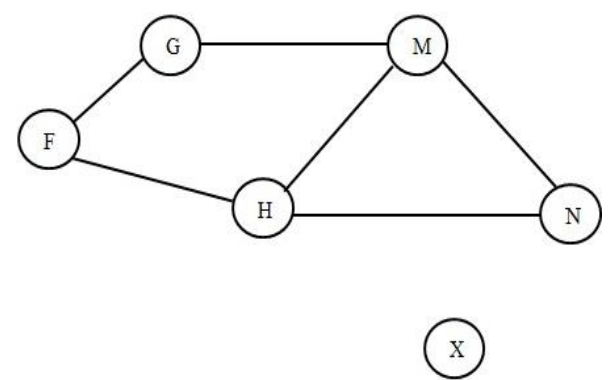

Fig. 5. Ad-hoc network for 6 nodes.

The structures of the master route cache by node $\mathrm{F}$ for the network in Fig. 4 are represented in Table II as fowling:

TABLE II: THE MASTER ROUTE CACHE STRUCTURE FOR NODE F

\begin{tabular}{lllll}
\hline \hline Source & Destination & $\begin{array}{l}\text { Number } \\
\text { of hops }\end{array}$ & Status & Route Inder \\
\hline F & G & 1 & 1 & 4 \\
F & H & 1 & 1 & 6 \\
F & H & 2 & 1 & 8 \\
F & H & 3 & 1 & 5 \\
F & N & 2 & 1 & 3 \\
F & N & 3 & 1 & 2 \\
F & N & 3 & 1 & 1 \\
F & N & 4 & 1 & 7 \\
F & M & 4 & 1 & 9 \\
F & M & 2 & 1 & 10 \\
F & M & 2 & 1 & 11 \\
F & X & 3 & 0 & 12 \\
\hline \hline
\end{tabular}

Note:

- Source represented to the source node.

- Destination represented the target of a route from source to destination.

- Number of hops represent the packet will be jumping from node to another to reach a destination.

- Status is represented the status of the route if was broken or not ( 0 /inactive- broken) (1/active).

- The route index represents the pointer for the route in the Index Route Cache.

The Structure of the Index route Cache by node $\mathrm{F}$ for the network was explained in Fig. 4 is represented in Table III as fowling:

TABLE III: THE INDEX ROUTE CACHE STRUCTURE OF NODE F

\begin{tabular}{ll}
\hline \hline The Route Index & Route \\
\hline 1 & F-G-H-N \\
2 & F-G-M-N \\
3 & F-H-N \\
4 & F-G \\
5 & F-G-M-H \\
6 & F-H \\
7 & F-G-M-H-N \\
8 & F-G-H \\
9 & F-G-M \\
10 & F-H-M \\
11 & F-G-H-M \\
12 & F-H-N-M \\
\hline \hline
\end{tabular}

Note:

In Table III Route Index: represented the index for the Route.

Route: represented the node that packets will pass through from the source node to the destination.

\section{2) Route discovery}

* Source node in the network has packet to send.

The source node checks its cache, for any available active routes.

A- If a route was available then will get a whole route from the second part of the cache then use it to send the packet.

$\mathrm{B}-*$ if the route not available so, will call to the procedure of route discovery.

a) Procedure one

The source node will broadcast Route Request (RREQ) message to all the nodes in its network. This message contains the source address and destination address.

b) Procedure tow

For any node in network range which receives the RREQ.

1) (YES). If this node was a destination node so, it will reverse the route then will send the Route Reply (RREP) to the source.

2) If (NO) mean this node was not destination then.

- If have a route to the destination then will append its route to available route and reply by RREP message.

- If this node were not having any route then it's just will add the address to the RREQ and then rebroadcast it.

- While the source node receives RREP, so it will check the route on its cache.

If (YES) mean, the route is already then will change its status to the 1 .

If (NO) mean the route was new or not exists in the cache then will:

1) Use the router order program to append the new route to Master-Route cache which dependent on the destination and the number of hops then update according to the route age.

2) Append the route to the Index Route Cache then uses the index as a pointer to the Master Route Cache, which the source node will use this route to send its packet.

3) The stage three: route maintenance

1) * any broken link if happened in the network cause a message will send to the source node by the intermediate nodes.

2) When the source node receives this broken link message it will:

- Deactivate this link broken on its master route cache.

- Check on the master route cache for any alternate route.

- If was available then will copy route from the index route cache to the packet then retransmit it.

- If was not available then will send RREQ message to a new route.

\section{4) Route status checker}

Check route status periodically to delete any inactive broken routes from Index Route Cache and master Route Cache shown as in below:

10- Start

20- Initial simulation environment then go to step 50

30- Initial cache node structure

40- periodically check for router statues

50- source nodes has packet to send 
60- Check node cache for active route if was available then Go to step140 if not then go to the next step

70- Send RREQ to neighbor node

80- Intermediate node receives RREQ then will check of its destination if yes then go to step 100 if No Go to next step 90 - has route to destination if yes add its route to the available route then go to step 70 if No then put its address and retransmit RREQ then go to step 70

100- Reverse route and send RREP to source

110- Source node check its cache for RREP

120- If the rout already (YES) update route status to 1 then GO to step 140 if NO then GO to next step

130- Add the new route to cache

140- Take route for second part of cache and use it for transition

150- Check the broken link if no found goes to step 180 if yes go to next step

160- Send RREP message to source

170 - Source deactivate route (to 0 ) then go to step 60

180- END

The all steps shows the whole processing for all four stages for enhance DSR cache routing protocol

The enhance DSR are represented in steps 30, 40, $60,110,120,130$ and 170 .

The steps 20 and 30 represented the initialization stage.

The steps 150, 160 and 170 represented the route maintenance stage.

The others steps represented the route discovery stage and checkers.

\section{NETWORK SIMULATION}

There is different performance metrics for DSR protocol and DSR Cache. We represented a scenario with 30 nodes with the following metrics:

Average delay: it refers to all the time, which is taken by packet to transmit across the network from source to the destination node which includes all delay caused through route discovery latency, retransmission of delays at MAC, transfer time and propagation. If the protocol shows higher end-to-end delay that means the performance of this protocol is unsuitable due to the network congestion [8].

Router discovery time: the time of discovering a route to a specific destination at the time when a route demand has sent out to discover a route to that destination until the route response is received with a route to this destination. In this statistic, the time to discover the path to a specific destination on all the nodes in the network will be represented.

Throughput: it shows the number of bits (bits/sec) forward and receives between nodes in an ad-hoc network. The throughput has referred as the ratio of the total amount data which are received from a sender to a time takes for the receiver to make a final packet [12]. The routing overhead represents the total number of routing packet transmission during the simulation whereas each transmission of the packet means each hop is counted as one transmission.

To implement simulation, wireless area network (WLAN) which consists of simple network entities as nodes (mobile) and base station was set up. The simulation consists of 30 nodes as shown in Table IV.
TABLE IV: THE SIMULATION PARAMETERS

\begin{tabular}{ll}
\hline \hline Parameters & \\
\hline Examiner Protocol & DSR protocol \\
Simulation Time & $600 \mathrm{sec}$ \\
Simulation Area & Campus $800 \mathrm{M} \times 800 \mathrm{M}$ \\
No. of Node s & 30 \\
Application configuration & Video conference Email for \\
& all nodes \\
Performance metric & Route discovery, delay \\
& average and throughput \\
Type of node & Mobile \\
Packet size & 512 bytes \\
PPP server & Configuration with all \\
& nodes in scenario \\
IP cloud & ip32_cloud \\
MANET Gateway & wlan_ethernet_slip4 \\
& Operate on IEEE802.11b \\
& standard \\
\hline \hline
\end{tabular}

The parameter for the entire scenario, including route discovery, average delay and throughput for both case in DSR routing protocol are used. The behaviors of these protocols in this scenario were analyzed in terms of network performance, and comparison results comparison was made. All the network nodes are modeled on area $800 \times 800 \mathrm{~m}$ under high network load. The Entity of the base station of this network communicates with nodes on the network.

\section{Simulation Results}

The simulation time set as $600 \mathrm{sec}$, with use of IEEE802.11 protocol for media access control (MAC) layer, the constant of bit Rate 5 packets/sec as the transmission rate. The application configuration for this scenario is video conference and email download and uploads. We selected two scenarios, the first one represented standard DSR, and the second represented the modified DSR. Then we measured the average delay and route discovery for new DSR.

Fig. 6 shows the effectiveness of the proposed algorithm that reduced the Delay, The reason that the cache in DSR protocol used to store all the route are learned from the source node to avoid unnecessary routing this will affect the performance of DSR protocol as well as the cache contain the topology of the network for every node.

Fig. 7 shows the route discovery time in DSR cache has less than the standard DSR as shown in Fig. 8. It's obvious that the old structure's route discovery increase continuously with higher cache size, but with the new structure that a route discovery was decreased.

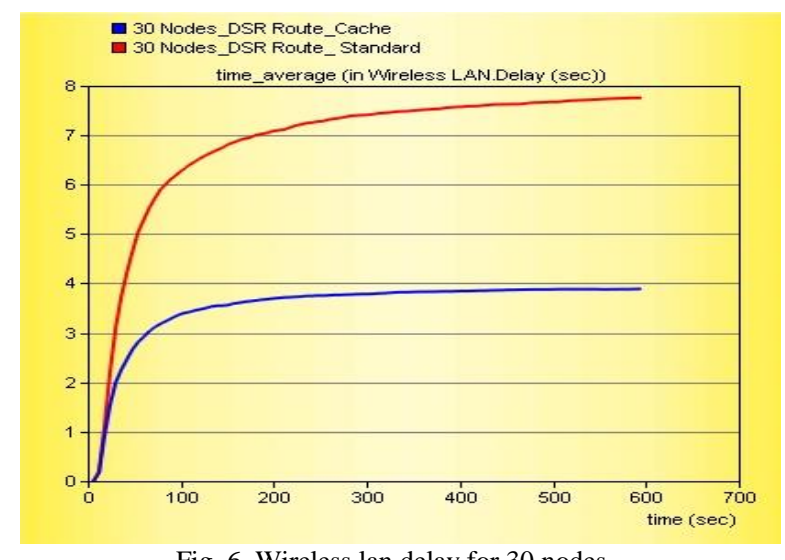

Fig. 6. Wireless lan delay for 30 nodes. 


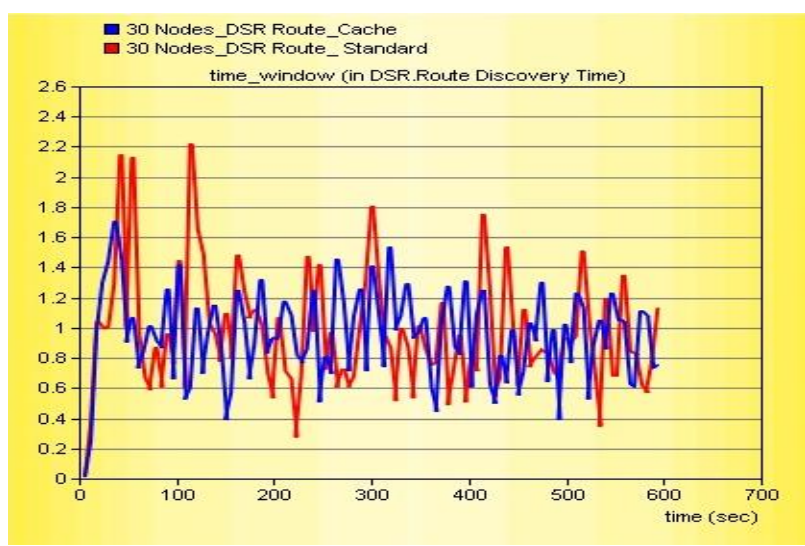

Fig. 7. Route discovery time for 30 nodes.

Fig. 8 shows that the proposed algorithm has good throughput for the network within 30 nodes.

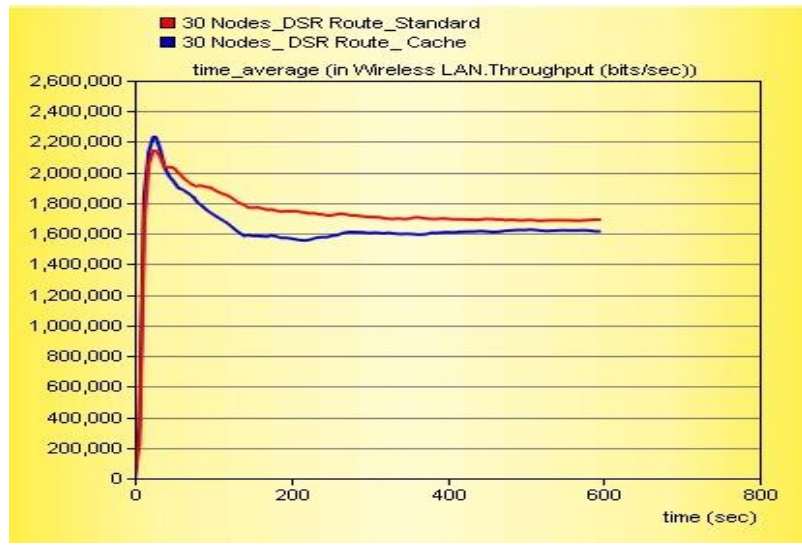

Fig. 8. Throughput for 30 nodes.

\section{CONCLUSION}

The proposed algorithm provides minimum delay and minimum time route discovery for both the Master and The index route cache , as well as the new route will be store in cache before the old ones, this will lead to decrease the use of old route,

It will probability increase packet reaches to the destination then solves the case of broken route problem. as well as, it will resolve the case of cache size problem by divided the cache route into two caches (Master and Index), the "Master cache route" it will be smaller than "Index Cache Route" which will store the complete route from the source to a destination node, but the "Master Cache Route" will store only the pointer of the master part. Eventually the proposed flowchart algorithm will enhance the route discovery and the time of delay and thus lead to enhance of DSR routing protocol.

\section{REFERENCES}

[1] J. Broch, D. B. Johnson, and D. A. Maltz, "The dynamic source routing protocol for mobile Ad-hoc network," Internet Draft, Carnegie Mellon University, pp. 4-6, 1998.

[2] B. David, A. David, and B. Josh, "DSR: The dynamic source routing protocol for multi-hop wireless Ad hoc networks," Carnegie Mellon University, pp. 1-25, 2001.

[3] Y. Hu, A. Perrig, and D. B. Johnson, "Packet leashes a defense against wormhole attacks in wireless networks," in Proc. Twenty-Second Annual Joint Conference of the IEEE Computer and Communications, vol. 3, pp. 1976-1986.
[4] N. A. Husieen, O. B. Ghazali, S. Hassan, and M. M. Kadhum, "Route cache update mechanisms in DSR protocol - A survey," in Proc. International Conference on Information and Network Technology, 2011, vol. 4, pp. 136-141.

[5] M. K. M. and S. R. Das, "Performance of route caching strategies in dynamic source routing," in Proc. the $2^{\text {nd }}$ Wireless Networking and Mobile Computing (WNMC), 2001, pp. 425-432.

[6] W. Lou and Y. Fang, "Predicative caching strategy for on- demand routing protocols in wireless Ad Hoc networks," Wireless Networks, vol. 8, no. 10, pp. 671-679, 2002.

[7] X. Yu, "A distributed adaptive cache update algorithm for the dynamic source routing protocol," IEEE Transactions on Mobile Computing, vol. 5, pp. 609-626, 2001.

[8] B. Y. He, C. S. Raghavendra, and S. Berson, "Active packets improve dynamic source routing for Ad Hoc networks," in Proc. IEEE Conference on Open Architectures and Network Programming, 2002.

[9] J. Chen, Y. Tang, D. Fu, and H. Chang, "On the improving strategies upon the route cache of DSR in MANET," Lecture Notes in Computer Science, vol. 6406, pp. 445-458, 2010.

[10] A. K. Dwivedi, S. Kushwaha, and O. P. Vyas, "Performance of routing protocols for mobile Ad hoc and wireless sensor networks: A comparative study," International Journal of Recent Trends in Engineering, vol. 2, no. 4, November 2009.

[11] K. Gorantala, "Routing protocols in mobile Ad-hoc networks," Master's Thesis, Umea University, 2006.

[12] M. N. Mohammad and N. Sulaiman, "A new broadcast algorithm to optimize routing protocol in mobile Ad hoc networks," Journal of Applied Sciences, vol. 13, pp. 588-594, 2013.

[13] C. Alaettinoglu, A. U. Shankar, K. Dussa-Zieger, and I. Matta, "Design and implementation of MaRS: A routing testbed," University of Maryland, pp. 1-31, 1993

[14] P. K. Maurya, G. Sharma, V. Sahu, A. Roberts, M. Srivastava, M. T. Scholar, and M. Allahabad, "An overview of AODV routing protocol," International Journal of Modern Engineering Research, vol. 2, no. 3, pp. 728-732, 2012

[15] L. Shrivastava, S. S. Bhadauria, and G. S. Tomar, "Performance evaluation of routing protocols in MANET with different traffic loads," in Proc. International Conference on Communication Systems and Network Technologies, pp. 13-16, 2011.

[16] M. Abolhasan, T. Wysocki, and E. Dutkiewicz, "A review of routing protocols for mobile ad hoc networks," Ad Hoc Networks, vol. 2, pp. 1-22, 2004.

[17] S. Doshi, S. Bhandare, and T. X. Brown, "An on-demand minimum energy routing protocol for a wireless ad hoc network," $A C M$ SIGMOBILE Mobile Computing and Communications Review, vol. 6 , no. 3, p. 50, Jun. 2002

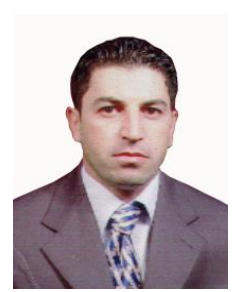

A. A. Azeez is currently a PhD candidate at Faculty of Computer Systems \& Software Engineering, University Malaysia Pahang (UMP). He has obtained bachelor degree from Technical College in Mosul, Department of Computer Engineering Technology in June 2000. After graduated he has been worked in many company service of internet, he currently work as an engineer in ministry communication of Iraq. He is going to recieve the master degree in compute science from University Malaysia Pahang in 2014. His research interests include the network security and mobile communication

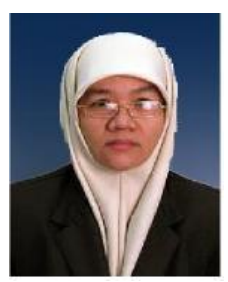

N. Sulaiman graduated from Sheffield Hallam University with a BSc (Hons) degree in computer studies in 1994. She worked with employment service in UK as a network support assistant and she involved in a research on Novell Netware. After graduation, she worked as a research officer at Artificial Intelligence System and Development Laboratory and involved in joint collaboration projects between the government of Malaysia and Japan for about 5 years. She completed her MSc degree in information technology and was involved in a research on Wireless Application Protocol (WAP). She obtained her PhD degree in mobile communication and networks from Newcastle University in UK. Currently, she is a senior lecturer at Faculty of Computer System and Software Engineering, University Malaysia Pahang. Her main research interests include heterogeneous networks, mobile communication networks and information security. 
M. N. Mohammed graduated from Al-Rafidain University College with a BSc degree in information systems computers science in 2003 , he worked as a programmer with the Ministry of Communications in Iraq for about 6 years. He had obtained his master degree in computer science from Faculty of Computer Systems \& Software Engineering, University Malaysia Pahang in 2011 and was involved in a research on network security. He obtained his $\mathrm{PhD}$ degree in mobile communication and wireless networks from University Malaysia Pahang in 2014. His main research interests include mobile communication and wireless networks, network security, computer information systems.

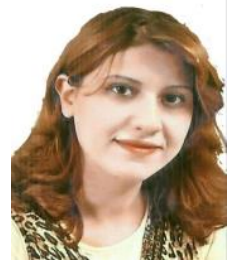

G. M. Abdulsaheb is currently a $\mathrm{PhD}$ student at Computer System and Software Engineering Department, University Malaysia Pahang (UMP)

Ghaidaa obtained her bachelor degree from Computer Engineering University of Technology in Iraq, with grade (very good) in 2007. After graduation she worked at Computer Engineering University of Technology, then she pursued her master study in Iraq at Al-Nahrain University, Faculty of Information Engineering and then obtained her master degree in 2012, Ghaidaa is a lecturer in University of Technology now, her main research interests include the mobile communication and wireless networks. 